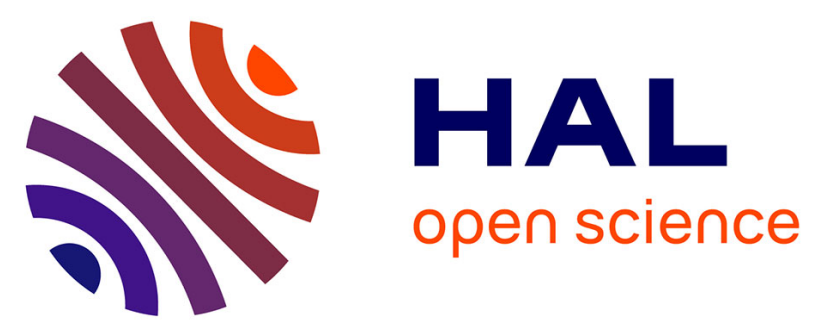

\title{
Residual fixed pattern noise and random telegraph signal noise of a MWIR T2SL focal plane array
}

Isabelle Ribet-Mohamed, Vignesh Arounassalame, Jean Nghiem, Marcel Caes, Maxence Guénin, Linda Höglund, Eric Costard, Philippe Christol

\section{- To cite this version:}

Isabelle Ribet-Mohamed, Vignesh Arounassalame, Jean Nghiem, Marcel Caes, Maxence Guénin, et al.. Residual fixed pattern noise and random telegraph signal noise of a MWIR T2SL focal plane array. Infrared Technology and Applications XLV, Apr 2019, Baltimore, France. pp.10.1117/12.2520211, 10.1117/12.2520211 . hal-02162731

\section{HAL Id: hal-02162731 \\ https://hal.science/hal-02162731}

Submitted on 30 Oct 2019

HAL is a multi-disciplinary open access archive for the deposit and dissemination of scientific research documents, whether they are published or not. The documents may come from teaching and research institutions in France or abroad, or from public or private research centers.
L'archive ouverte pluridisciplinaire HAL, est destinée au dépôt et à la diffusion de documents scientifiques de niveau recherche, publiés ou non, émanant des établissements d'enseignement et de recherche français ou étrangers, des laboratoires publics ou privés. 


\section{Residual fixed pattern noise and random telegraph signal noise of a MWIR T2SL focal plane array}

I. Ribet-Mohamed, V. Arounassalame, J. Nghiem, M. Caes, M. Guénin, et al.

I. Ribet-Mohamed, V. Arounassalame, J. Nghiem, M. Caes, M. Guénin, L. Höglund, E. Costard, P. Christol, "Residual fixed pattern noise and random telegraph signal noise of a MWIR T2SL focal plane array ," Proc. SPIE 11002, Infrared Technology and Applications XLV, 110020E (7 May 2019); doi: $10.1117 / 12.2520211$

Event: SPIE Defense + Commercial Sensing, 2019, Baltimore, Maryland, United States 


\title{
Residual fixed pattern noise and random telegraph signal noise of a MWIR T2SL focal plane array
}

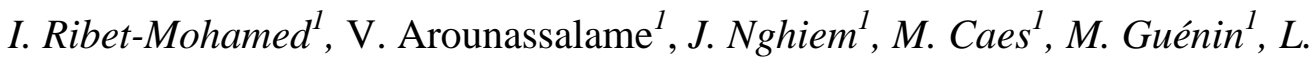 \\ Höglund $^{2}$, E. Costard ${ }^{2}$, P. Christol ${ }^{3}$ \\ ${ }^{1}$ ONERA, Chemin de la Hunière, F- 91761 Palaiseau, France. \\ ${ }^{2}$ IRnova AB, Electrum 236, SE-164 40 Kista, Sweden \\ ${ }^{3}$ IES, Univ. Montpellier, CNRS, F-34000 Montpellier, France
}

\begin{abstract}
Stability over time has recently become a figure of merit of major importance to compare the performances of infrared focal plane arrays (FPA) of different technologies. Indeed, this parameter dictates how often the calibration of operational electro-optical systems has to be done, and thus reflects the availability of the system during an operational mission.

The stability over time is generally estimated through fixed pattern noise (FPN) and residual fixed pattern noise (RFPN) measurements after a two-point correction. However, each laboratory or industrial has its own protocols and criteria, such that published results cannot be easily compared. Recent studies also showed that random telegraph signal (RTS) noise, which leads to flickering pixels, can strongly affect the image quality, so the question arises as to wether these RTS pixels have an effect on RFPN.

In this paper, we describe our experimental protocol to evaluate the stability over time of an FPA and to count up / classify flickering pixels. We then present the results obtained on a T2SL MWIR Integrated Detector Dewar Cooler Assembly (IDDCA) provided by IRnova. Our measurements show that the stability over time of the T2SL MWIR IDDCA are excellent: first, in terms of FPN/RFPN; then, in terms of RTS noise with only a few blinking pixels. We also show that the RTS pixels having an effect on the RFPN are fully detected by the algorithm used to rule out defective pixels before calculating RFPN.
\end{abstract}

Keywords: stability over time, FPN, RFPN, correctability, T2SL, MWIR, FPA

*Corresponding author: Tel.: (+33) 180386 362; e-mail address: isabelle.ribet@ onera.fr

Infrared Technology and Applications XLV, edited by Bjørn F. Andresen,

Gabor F. Fulop, Charles M. Hanson, Proc. of SPIE Vol. 11002, 110020E

(c) 2019 SPIE · CCC code: $0277-786$ X/19/\$18 $\cdot$ doi: 10.1117/12.2520211 


\section{Introduction}

Type-II Superlattice (T2SL) detectors are a recent commercial infrared technology designed to meet high-performance imaging applications [1-10], such that electro-optical characterisation efforts are now focused on system-oriented merit functions, for example MTF (Modulation Transfer Function) or stability over time. MTF describes how well a detector can reproduce spatial frequencies [11], whereas stability over time dictates how often the calibration of operational electro-optical systems has to be done, and thus reflects the availability of the system during an operational mission.

The stability over time is generally estimated through fixed pattern noise (FPN) and residual fixed pattern noise (RFPN) measurements, the latter being evaluated after a linear two-point calibration (TPC). However, each laboratory or industrial has its own protocol and criteria to exclude some pixels considered as defective, such that published results cannot be easily compared. Moreover, one can wonder wether FPN and RFPN are affected by pixels with random telegraph signal (RTS) noise. The signal delivered by these pixels can oscillate between two (or more) levels at certain times, creating a particularly harmful blinking effect for image quality. Since the blinking is not permanent, detecting these pixels is a very difficult task.

In this paper, we describe our experimental protocol to evaluate the temporal stability of a FPA. We then present the results of long-term measurement campaigns realized on an InAs/GaSb T2SL focal plane array. We chose to carry out our measurements on a detector integrated in an operational packaging (IDDCA, which means Integrated Detector Dewar Cooler Assembly) in order to remain as close as possible to the real conditions of use. More precisely, our measurements were realized on a T2SL MWIR 320x256 pixels IDDCA provided by IRnova, on which we have already published very encouraging first stability over time measurements [12].

The first paragraph of this paper is dedicated to residual fixed pattern noise (RFPN) measurements. After recalling the principle of this measurement, we present our experimental setup and the data processing. Results obtained during a long-term measurement campaign are then reported and commented. The second paragraph focuses on random telegraph signal (RTS) noise, and presents the experimental setup, the data processing and the experimental results.

\section{Residual fixed pattern noise measurements}

The stability over time is generally estimated through fixed pattern noise (FPN). The FPN corresponds to the spatial fluctuations of the signal delivered by the pixels of a focal 
plane array (FPA) when it receives a homogeneous incident power. The fixed pattern noise can originate in the pixel itself (for example if there are differences in cut-off wavelengths or variations in active area thickness through the focal plane array) or in the read-out integrated circuit (for example if the output amplifiers have slightly different characteristics (gain, offset)). Non-uniformity correction (NUC) is generally realized with a linear two-point calibration [13]. To implement it, two uniform backgrounds are presented to the FPA (generated for example with two extended blackbodies at different temperatures). Assuming that the signal delivered by each pixel varies linearly with the incident power, one can calculate, for each pixel, a gain correction coefficient $\mathrm{G}_{\mathrm{i}, \mathrm{j}}$ and an offset correction coefficient $\mathrm{O}_{\mathrm{i}, \mathrm{j}}$, accounting for multiplicative and additive non-uniformities, respectively. They are defined as :

$$
\begin{gathered}
G_{i, j}=\frac{S_{i, j}\left(\phi_{1}\right)-S_{i, j}\left(\phi_{2}\right)}{\left\langle S_{i, j}\left(\phi_{1}\right)\right\rangle-\left\langle S_{i, j}\left(\phi_{2}\right)\right\rangle} \\
O_{i, j}=S_{i, j}\left(\phi_{1}\right)-G_{i, j} \cdot\left\langle S_{i, j}\left(\phi_{1}\right)\right\rangle
\end{gathered}
$$

where $S_{i, j}\left(\phi_{1}\right)$ (resp. $S_{i, j}\left(\phi_{2}\right)$ ) is the signal delivered by the pixel (i,j) for an incident power equal to $\phi_{1}$ (resp. $\left.\phi_{2}\right)$ and after removing the ROIC offset ; $\left\langle\mathrm{S}_{\mathrm{i}, \mathrm{j}}\left(\phi_{1}\right)\right\rangle\left(\operatorname{resp} .\left\langle\mathrm{S}_{\mathrm{i}, \mathrm{j}}\left(\phi_{2}\right)\right\rangle\right)$ is the spatially averaged signal delivered by the FPA (after removing the ROIC offset) for an incident power equal to $\phi_{1}$ (resp. $\left.\phi_{2}\right)$.

After 2 points correction, the signal delivered by the pixel (i,j) becomes:

$$
S_{i, j}^{\prime}(\phi)=\frac{S_{i, j}(\phi)-O_{i, j}}{G_{i, j}}
$$

When applying the two-point correction that has just been calculated, the corrected image is perfectly uniform at an incident power equal to $\phi_{1}$ or $\phi_{2}$.

The two-point calibration has the advantage of being easy to implement and very effective at the time it is calculated. But the question is how long this calibration remains valid. To answer this question, the fixed spatial noise that persists after the two-point correction - called residual fixed spatial noise (RFPN) - is evaluated:

$$
\operatorname{RFPN}(\phi)=\sqrt{\frac{1}{N} \cdot \sum_{i} \sum_{j}\left(S_{i, j}(\phi)-\left\langle S_{i, j}(\phi)\right\rangle\right)^{2}}
$$

where $\mathrm{N}$ is the total number of pixels of the FPA.

To evaluate the performance of a system, temporal noise and residual fixed pattern noise are generally summed quadratically[14], the former being defined as the temporal 
fluctuations of the signal delivered by a pixel. Consequently, the influence of spatial noise decreases rapidly as soon as the RFPN is lower than the temporal noise; therefore, one can consider that the calibration is valid as long as the RFPN remains lower than the temporal noise, even if stricter criteria have been proposed for particular conditions [15] (with a human observer in the loop).

\section{Experimental setup}

The measuring bench relies on an extended area blackbody which is placed in front of the IDDCA. Scanning the blackbody temperature allows to scan the incident power on the

focal plane array. For each blackbody temperature explored, a 256-image cube is recorded at an integration time of $4 \mathrm{~ms}$, and another one at null integration time to measure the read-out integrated circuit (ROIC) offset.

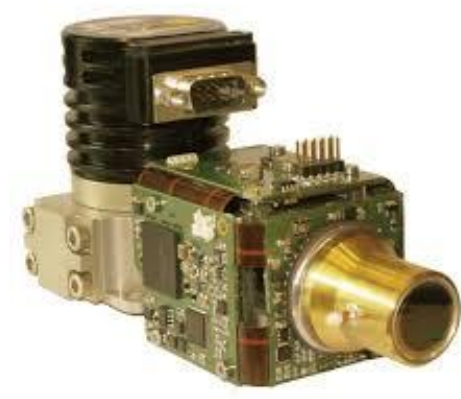

Figure 1 - T2SL MWIR IDDCA from IRnova.

\section{Data processing}

The analysis of the measurements consists of three main stages:

$>$ the detection of defective pixels, and once these have been set aside,

$>$ the application of the two-point correction

$>$ the calculation of the residual fixed pattern noise

In order to highlight the importance of the criteria used to define a defective pixel, we compared several algorithms. The first one, which will be further referred as the reference algorithm, considers the average image recorded for a $50 \%$ well fill and corrects it for the dome effect due to variation in the geometrical throughput between the center and the corner of the focal plane array. It then sets aside pixels with continuous level, noise (defined as the standard deviation of the signal of one pixel over 256 images), responsivity or noise equivalent temperature difference (NETD) too far from the average values. The exact criteria are summarized in Table 1, together with the number of pixels falling into each category. The total number of defective pixels detected with this algorithm is 41 , which corresponds to an 
operability of $99.95 \%$. As can be seen in Figure 2, the spatial distribution of the defective pixels is relatively homogeneous, with only 3 clusters with $2 \times 1$ pixels, and no bigger cluster.

\begin{tabular}{|c|c|c|}
\hline Continuous level & Criteria & Number of pixels \\
\hline Noise & $\left|S_{i, j}-\left\langle S_{i, j}\right\rangle\right|>30 \% \cdot\left\langle S_{i, j}\right\rangle$ & 31 \\
\hline Responsivity & $\left|\sigma_{i, j}-\left\langle\sigma_{i, j}\right\rangle\right|>50 \% \cdot\left\langle\sigma_{i, j}\right\rangle$ & 25 \\
\hline NETD & $\left|N E T D_{i, j}-\left\langle N E T D_{i, j}\right\rangle\right|>30 \% \cdot\left\langle R_{i, j}\right\rangle$ & 34 \\
\hline Total & & 37 \\
\hline
\end{tabular}

Table 1 - Criteria used to classify one pixel as a defective one (reference algorithm). $S_{\mathrm{i}, \mathrm{j}}$ is the signal delivered by the pixel, $\left\langle S_{i, j}\right\rangle$ is the spatially averaged signal, $\sigma_{i, j}$ is the noise of the pixel, $\left\langle\sigma_{i, j}\right\rangle$ is the spatially averaged noise, $R_{i, j}$ is the responsivity of the pixel, $\left\langle R_{i, j}\right\rangle$ is the spatially averaged responsivity, NETD $D_{i, j}$ is the NETD of the pixel and $\left\langle N E T D_{i, j}\right\rangle$ the spatially averaged NETD. The total number of defective pixels is lower than the sum of the number of pixels sorted as defective with only one criterion, since most of the defective pixels fall into more than one category.

The second algorithm, which we will call advanced algorithm, is based on the same principle, except that a two-point correction (calculated from images at 33\% and 66\% well fill) is applied after the dome effect is corrected and before the defective pixels are detected. The exact criteria are summarized in Table 2, together with the number of pixels falling into each category. The total number of defective pixels detected with this algorithm is 49, which corresponds to an operability of $99.94 \%$. As can be seen on the defective pixels map presented in Figure 2, the spatial repartition of defective pixels is once again quite homogeneous.

\begin{tabular}{|c|c|c|}
\hline & Criteria & Number of pixels \\
\hline Continuous level & $\left|S_{i, j}-\left\langle S_{i, j}\right\rangle\right|>10 \cdot\left\langle\sigma_{i, j}\right\rangle$ & 43 \\
\hline Noise & $\left|\sigma_{i, j}-\left\langle\sigma_{i, j}\right\rangle\right|>50 \% \cdot\left\langle\sigma_{i, j}\right\rangle$ & 25 \\
\hline Responsivity & $\left|R_{i, j}-\left\langle R_{i, j}\right\rangle\right|>30 \% \cdot\left\langle R_{i, j}\right\rangle$ & 34 \\
\hline NETD & $\left|N E T D_{i, j}-\left\langle N E T D_{i, j}\right\rangle\right|>100 \% \cdot\left\langle N E T D_{i, j}\right\rangle$ & 37 \\
\hline Total & & 49 \\
\hline
\end{tabular}

Table 2 - Criteria used to classify one pixel as a defective one (advanced algorithm). 


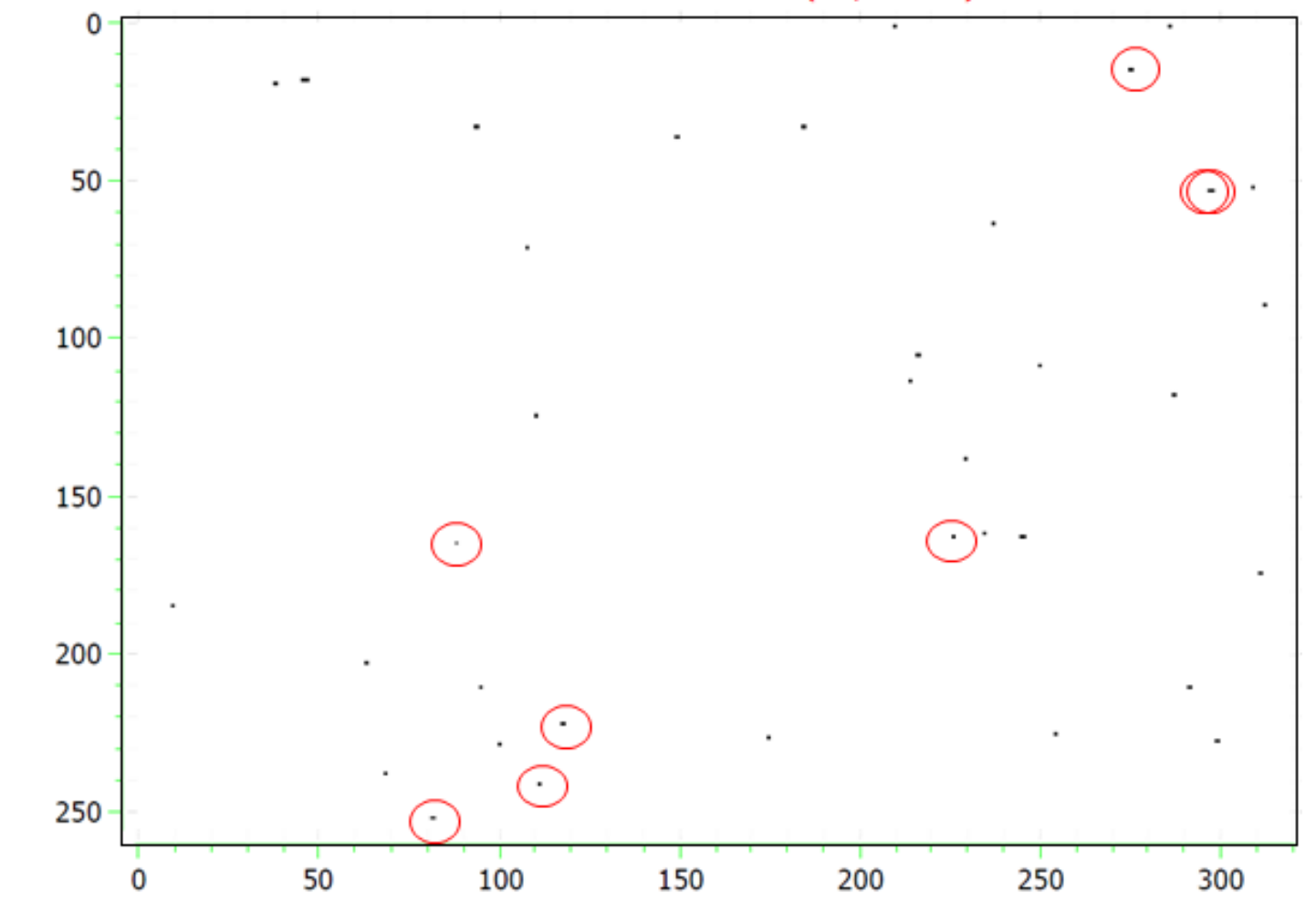

Figure 2 -Defective pixels map detected with the reference algorithm (black dots) and by the advanced algorithm

(black dots + red circles). 41 (resp 49) pixels are set aside with the reference (resp advanced) algorithm, which represents an operability of 99.95 (resp $99.94 \%)$.

Once the defective pixels have been set aside, we apply the gain and offset corrections (see equation (3)) and then calculate the residual fixed pattern noise thanks to equation (4). Results are presented in the following paragraph.

\section{Results and discussion}

Figure 3 presents the ratio between the residual fixed pattern noise and the temporal noise (TN) as a function of the well fill factor. The left-hand side (resp. right-hand side) graph corresponds to the case where defective pixels are detected with the reference algorithm (resp. advanced algorithm). In both cases, the gain and offset corrections were calculated on the first day of the campaign. The RFPN/TN results obtained that day exhibit the expected $\mathrm{W}$-shape, reaching zero for the two values of the power used to calculate the gain and offset coefficients. The RFPN/TN measurements obtained on the following days are all higher than those obtained on the first day, but if we trace their temporal evolution (see Figure 4), it appears that the RFPN is remarkably stable over 6 weeks. More precisely, the ratio between the RFPN and the temporal noise is typically equal to 1.1 for the reference algorithm and equal to 0.83 for the advanced algorithm. 

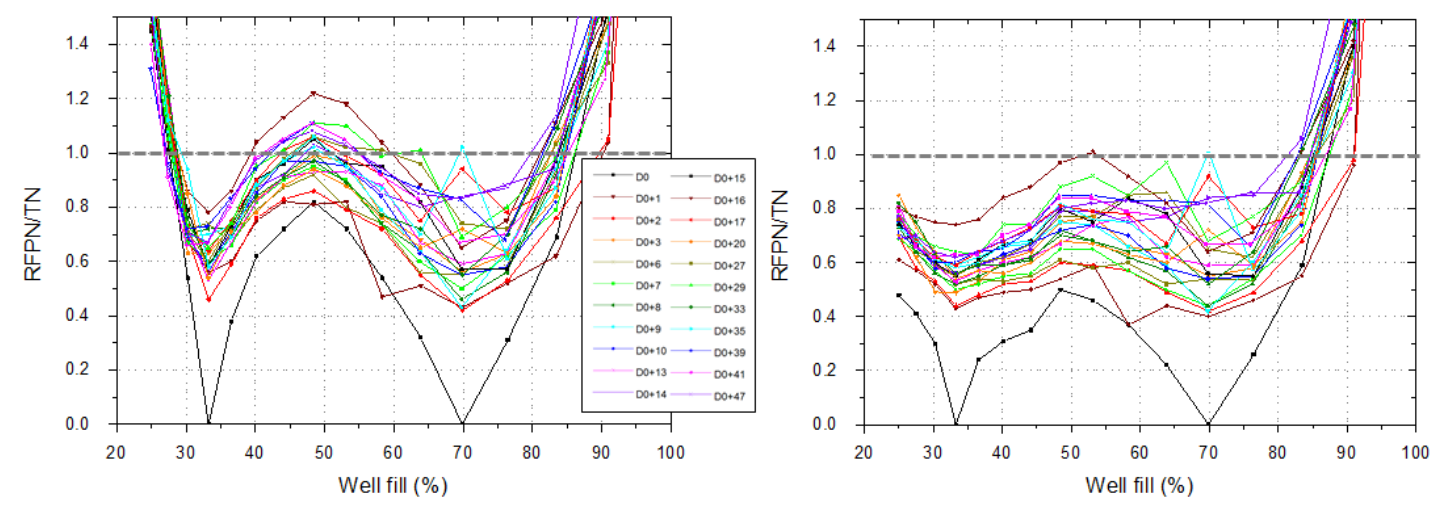

Figure 3 - Ratio between residual fixed pattern noise (RFPN) and temporal noise (TN) as a function of well fill, for measurements realized during 7 weeks. Left : 41 defective pixels (identified with the reference algorithm) were set aside ; Right : 49 defective pixels (identified with the improved algorithm) were set aside. The gain and offset coefficient used in the two-point corrections are those calculated on the first day of the campaign.

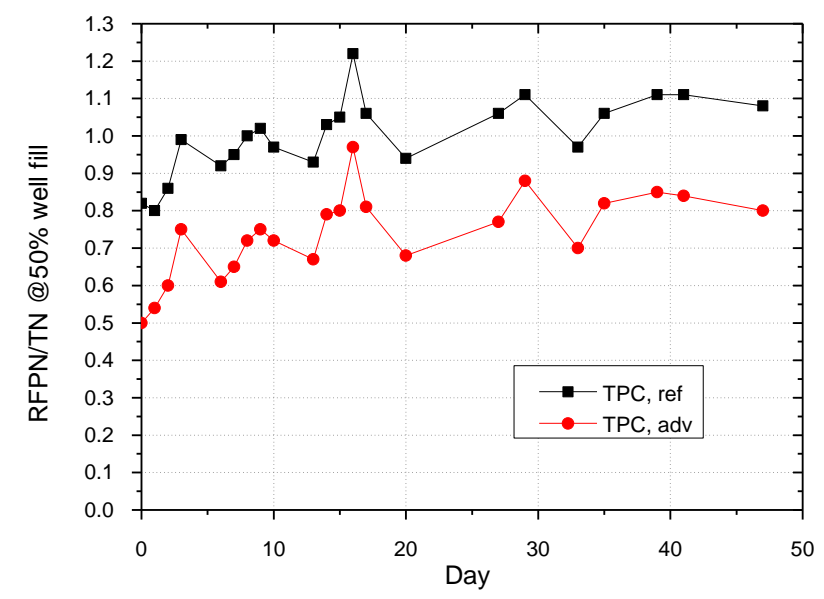

Figure 4 - Temporal evolution of the ratio between the residual fixed pattern noise and the temporal noise at $50 \%$ well fill, using the reference algorithm or the advanced one to detect defective pixels. The gain and offset coefficient used in the two-point corrections are those calculated on the first day of the campaign.

These results clearly show that the temporal stability of the T2SL MWIR FPA is excellent. They also stress the importance of the data processing phase and the criteria used to rule out defective pixels, since removing 8 more pixels decreases the RFPN/TN plateau of $25 \%$. It appeared that these 8 pixels presented a signal close to the average for $50 \%$ well fill (and were therefore not detected with the reference algorithm), but that they were strongly non-linear and impossible to correct with a TPC calibration. That's why the second algorithm detected them. 


\section{Random telegraph signal noise study}

Pixels are considered as exhibiting random telegraph signal (RTS) noise when their signal oscillates between at least two levels, while the received power and operating conditions do not change. RTS noise can be particularly detrimental to image quality since the resulting blinking effect cannot be suppressed by 2-point correction. It is thus considered as a major challenge that different technologies will have to face [16-18], especially in addressing high operating (HOT) and size, weight and power (SWaP) requirements.

\section{Experimental setup and data processing}

Studying RTS noise requires long temporal acquisitions under fixed conditions (integration time, blackbody temperature...). We determined that recording cubes of 5000 images was a good compromise between RTS pixels detection efficiency and data processing complexity. The integration time was set to $4 \mathrm{~ms}$. In order to study the effect of RTS pixels on RFPN, we needed simultaneous RFPN measurements so we scanned the blackbody temperature between $10^{\circ} \mathrm{C}$ and $58^{\circ} \mathrm{C}$ by steps of $3^{\circ} \mathrm{C}$, and each time recorded a 5000 images data cube. Such RFPN/RTS measurements were repeated 24 times, representing a cumulated acquisition time of more than 9 hours. Some of the measurements were made within the same cooling, others after allowing the detector to return to room temperature.

To detect RTS pixels, we used an algorithm which starts by isolating those with excess temporal noise. Among these candidates, it then fits the histogram with one or several Gaussian functions: if two or more Gaussian functions are required, the pixel is considered as affected by RTS noise (see example Figure 5).
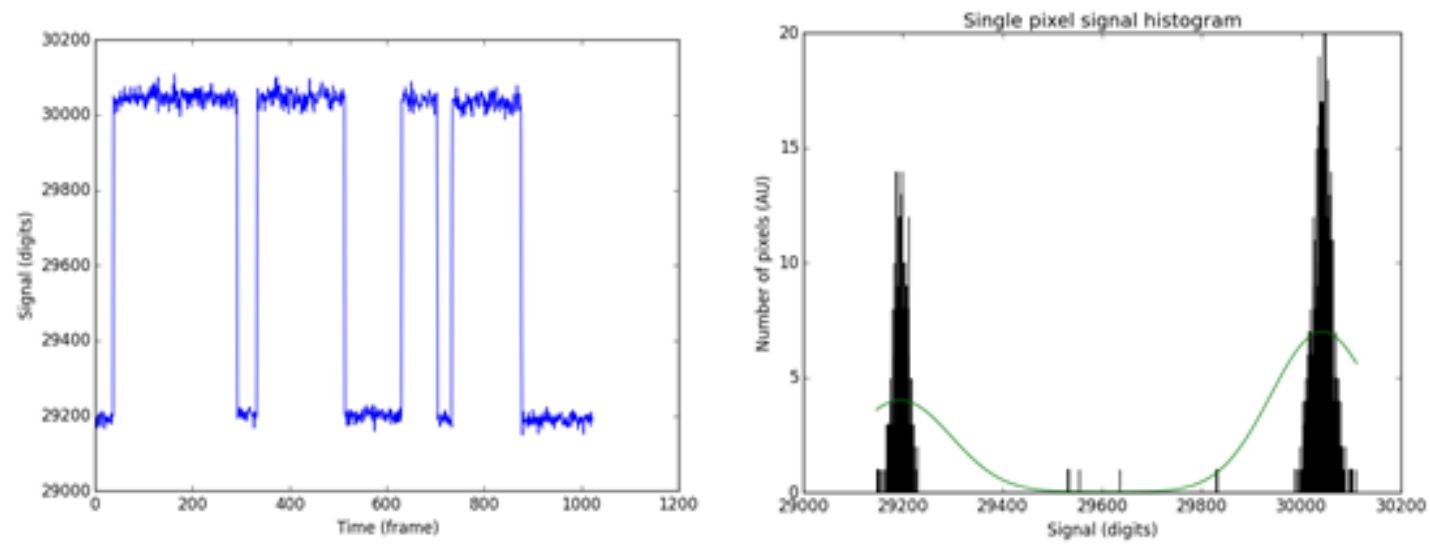

Figure 5 - Left : signal delivered by a pixel affected by a 2-level RTS noise as a function of time ; Right : associated histogram, showing two distinct populations.

\section{Results and discussion}

Our measurements show that the number of RTS pixels of the MWIR T2SL 320x256 pixels FPA is very low. It varies between 0 and 10 pixels detected by acquisition of 5000 
images (with a mean value of 6 RTS pixel per cube (acquired in 80 seconds). Table 3 presents a classification of the 52 RTS pixels detected on 192 cubes (for each RFPN measurement, only cubes corresponding to blackbody temperatures between $10^{\circ} \mathrm{C}$ and $31^{\circ} \mathrm{C}$ were considered, to ensure that RTS pixels would not be hidden by an increasing photon noise). Only 19 pixels have a true 2-level behaviour (see Figure $5 \mathrm{a}$ and b). 15 pixels exhibit spikes (see Figure $5 \mathrm{c}$ ). To finish with, 18 pixels exhibited low frequency temporal fluctuations that were considered as RTS noise (see Figure 5 d).

\begin{tabular}{|l|l|}
\hline 2 level-RTS pixels & 19 \\
\hline Spikes & 15 \\
\hline Atypical (low frequency fluctuations) & 18 \\
\hline Table 3 - Classification of pixels detected as affected by RTS noise.
\end{tabular}
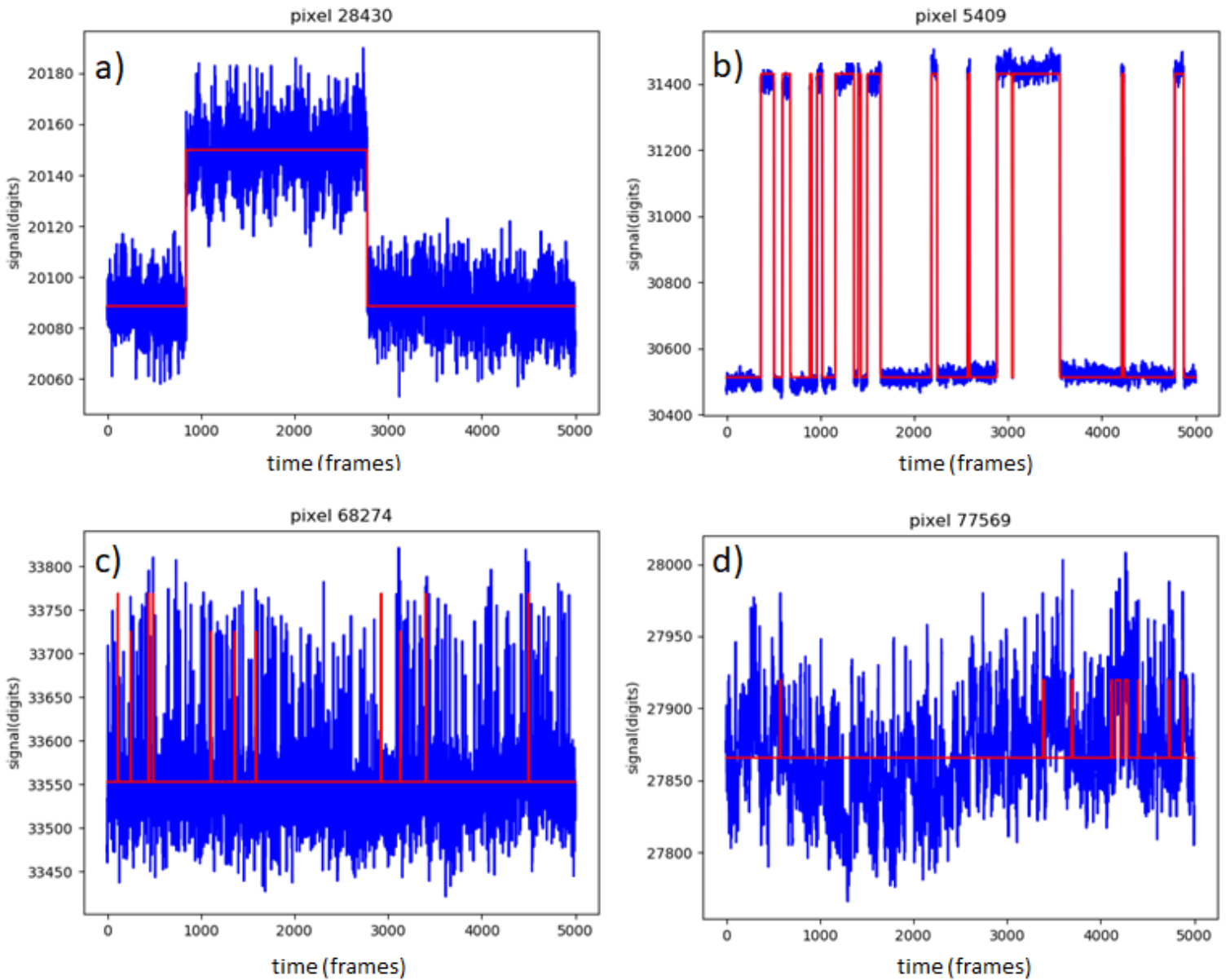

Table 4 - Signal as a function of time (one frame is taken each $16.7 \mathrm{~ms}$ ) for pixels detected as RTS : a) and b) exhibit a clear 2 level behavior, whereas c) exhibits spikes and d) exhibits low frequency fluctuations.

Let's now focus on the 19 pixels exhibiting a clear 2 level-behavior. 15 pixels out of them flicker less than $10 \%$ of the time (for example, pixel $n^{\circ} 28430$, shown in Figure 5 a, only flickers $2 \%$ of the time), 3 pixels flickers between $15 \%$ and $20 \%$ of the time and one pixel $\left(n^{\circ} 5409\right.$, see Figure 5 b) flickers $90 \%$ of the time. 
We also plotted in Figure 6 the instantaneous and the cumulative number of RTS pixels detected as a function of the measurement number. The cumulative number of RTS pixels increases more rapidly at the beginning of the curve and less rapidly for measurement numbers $>125$, which could be compatible with a stabilisation of the cumulative number of RTS pixels but the stabilisation regime is still not reached after 192 measurements $(\sim 4.5$ hours). Figure 6 also shows that the increase of the total number of RTS pixels is not correlated with new cooling cycles (materialized by arrows).

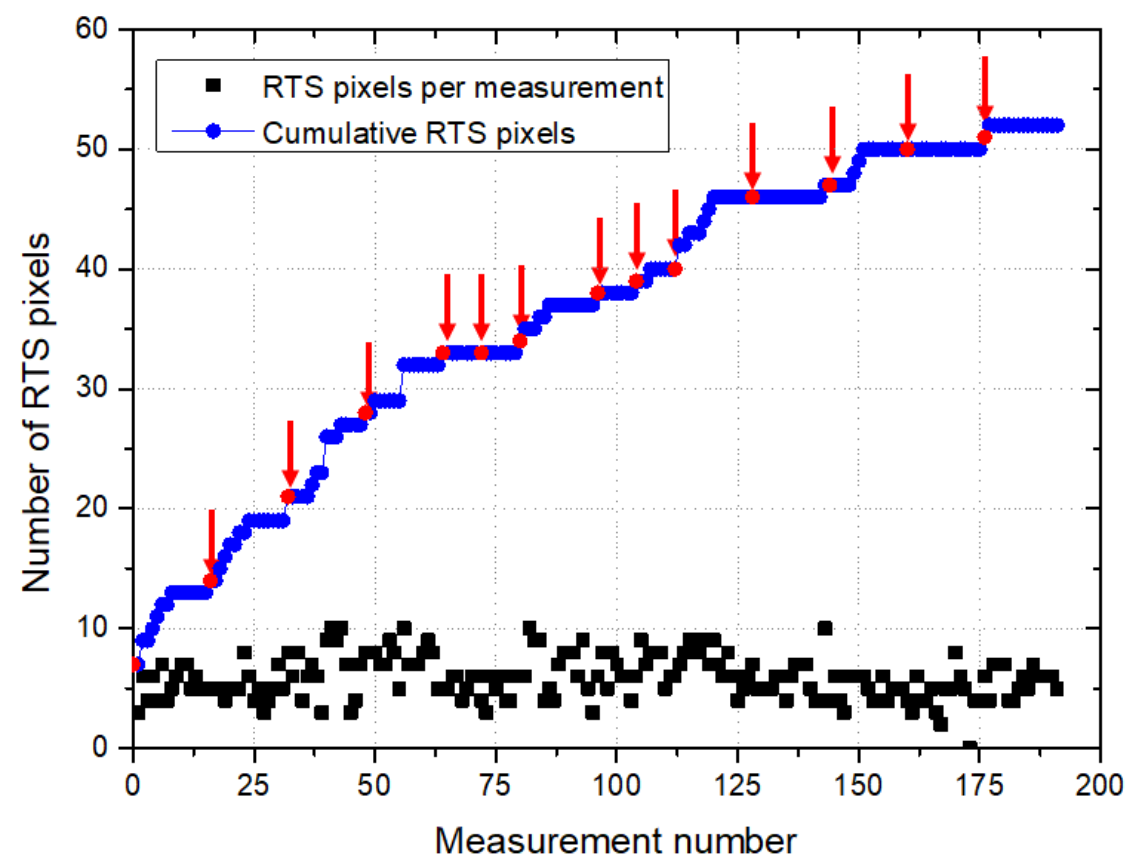

Figure 6 - Instantaneous and cumulative number of RTS pixels detected as a function of the measurement number. Red arrows indicate new cooling cycles.

Finally, we wondered whether the RTS pixels could affect the RFPN. From the data cubes acquired at different blackbody temperatures, we extracted RFPN measurements using the protocol described above. We first ruled out the 53 pixels considered as defective by our "advanced" algorithm (including 10 RTS pixels) and computed the RFPN/TN ratio as a function of the well fill, see Figure 7). We then also ruled out the 6 other RTS pixels which were not detected as defective, and computed the RFPN/TN ratio. Both curves superimpose, which proves that the RTS pixels which affect the RFPN were already detected by our "advanced" algorithm : there is no need to improve it. 


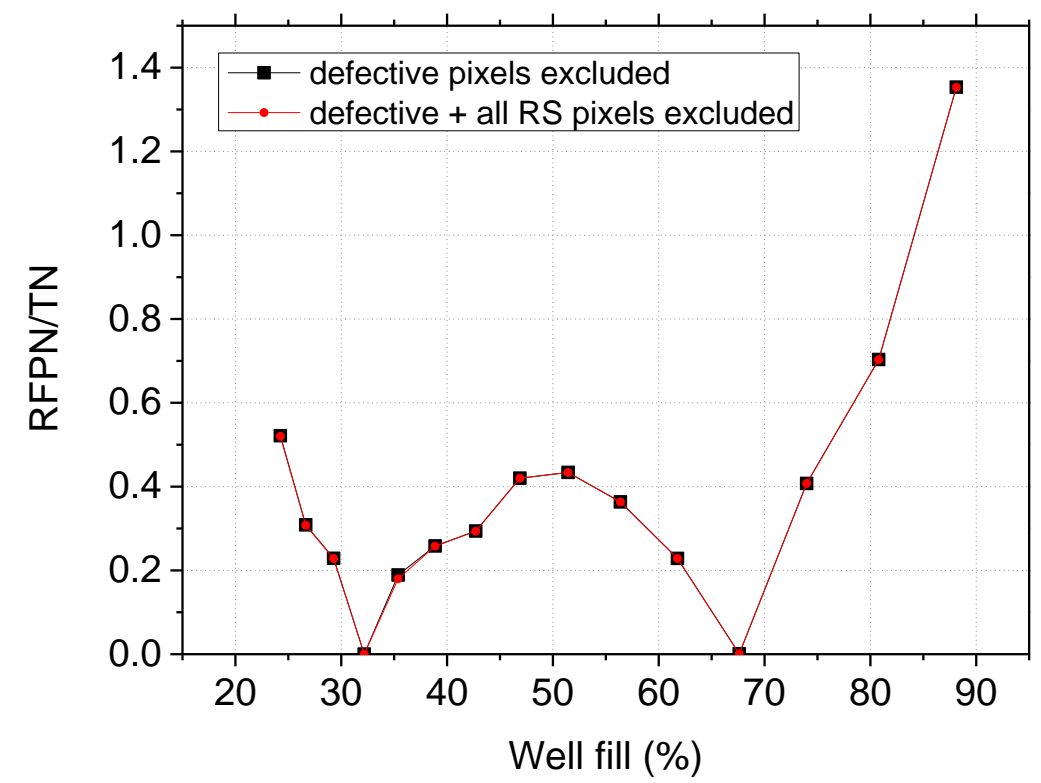

Figure 7 - Ratio between residual fixed pattern noise (RFPN) and temporal noise (TN) as a function of well fill, ruling out the defective pixels detected by our "advanced" algorithm (black) or these defective pixels and all the RTS pixels. The fact that the curves are superimposed proves that the RTS pixels that have an influence on the RFPN are all detected by our "advanced" algorithm.

\section{Conclusion}

In this paper, we described our experimental protocol to evaluate the stability over time of an FPA and to count up / classify pixels with random telegraph signal noise. We presented the results obtained for a T2SL MWIR 320x256 pixels IDDCA provided by IRnova. The stability over time is excellent over seven weeks. We stressed out the importance of the criteria used to rule out defective pixels. The RFPN/TN plateau can thus be decreased from 1.1 (with reference defective pixels detection and basic two-point correction (TPC)) to 0.83 (with advanced defective pixel detection and basic TPC).

Our measurements also allowed us to count up and classify pixels exhibiting a random telegraph signal (RTS) noise. The total number of 2-level RTS pixels is 19, for a 4.55 hours cumulative acquisition. 15 out of these 19 2-level RTS pixels flicker less than $10 \%$ of the time. We also showed that among the RTS pixels, those affecting the RFPN were already detected as defective by our "advanced" algorithm. 


\section{References}

[1] A.K. Sood, J.W. Zeller, R.E. Welser, Y.R. Puri, N.K. Dhar, P.S. Wijewarnasuriya, S. Krishna, Design and Development of Two-Dimensional Strained Layer Superlattice (SLS) Detector Arrays for IR Applications, Two-dimensional Materials for Photodetector Pramoda Kumar Nayak, IntechOpen (2018).

[2] A. Rogalski, M. Kopytko, P. Martyniuk, InAs/GaSb type-II superlattice infrared detectors: three decades of development, Proc. SPIE 10177 (2017) 1017715.

[3] R. Chevallier, A. Haddadi, M. Razeghi, Dark current reduction in microjunction-based compound electron barrier type-II InAs/InAs1-xSbx superlattice-based long-wavelength infrared photodetectors, Proc. SPIE 10540 (2018) 1054007.

[4] D.Z. Ting, A. Soibel, A. Khoshakhlagh, L. Höglund, S.A. Keo, S.B. Rafol, C.J. Hill, A.M. Fisher, E.M. Luong, J. Nguyen, J.K. Liu, J.M. Mumolo, B.J. Pepper, S.D. Gunapala, Antimonide type-II superlattice barrier infrared detectors, Proc. SPIE 10177 (2017) 101770N.

[5] P.C. Klipstein, E. Avnon, Y. Benny, A. Fraenkel, A. Glozman, E. Hojman, E. Ilan,

E. Kahanov, O. Klin, L. Krasovitski, L. Langof, I. Lukomsky, M. Nitzani, L. Shkedy,

I. Shtrichman, N. Snapi, R. Talmor, A. Tuito, S. Vaserman, and E. Weiss, Long Wave Infrared Type II Superlattice Focal Plane Array Detector, Defence Science Journal 67 (2017) 135.

[6] L. Höglund, C. Asplund, R. Marcks von Würtemberg, H. Kataria, A. Gamfeldt, S. Smuk, H. Martijn, E. Costard, Manufacturability of type-II InAs/GaSb superlattice detectors for infrared imaging, Infrared Physics \& Technology 84 (2017) 28.

[7] Q. Durlin, J.P. Perez, R. Rossignol, J.B. Rodriguez, L. Cerutti, B. Delacourt, J. Rothman, C. Cervera, P. Christol, InAs/InAsSb superlattice structure tailored for detection of the full midwave infrared spectral domain, Proc. SPIE 10111 (2017) 1011112.

[8] V. Daumer, V. Gramich, R. Müller, J. Schmidt, F. Rutz, T. Stadelmann, A. Wörl, R. Rehm, Photodetector development at Fraunhofer IAF: From LWIR to SWIR operating from cryogenic close to room temperature, Proc. SPIE 10177 (2017) 1017711.

[9] A. Kazemi, S. Myers, Z.Taghipour, S. Mathews, T. Schuler-Sandy, S. Lee, V. M. Cowan, E. Garduno, E. Steenbergen, C. Morath, G. Ariyawansa, J. Scheihing, S. Krishna, Mid-wavelength infrared unipolar nBp superlattice photodetector, Infrared Physics \& Technology 88 (2018) 114. [10] B. Tan, C. Zhang, W. Zhou, X. Yang, G. Wang, Y. Li, Y. Ding, Z. Zhang, H. Lei, W. Liu, Y. Du, L. Zhang, B. Liu, L. Wang, L. Huang, The $640 \times 512$ LWIR type-II superlattice detectors operating at 110 K, Infrared Physics \& Technology 89 (2018) 168.

[11] J. Nghiem, J. Jaeck, J. Primot, C. Coudrain, S. Derelle, E. Huard, M. Caes, S. Bernhardt, R. Haidar, P. Christol, I. Ribet-Mohamed, MTF measurements of a type-II superlattice infrared focal plane array sealed in a cryocooler, Opt. Express 26 (2018) 11034-11045.

[12] J. Nghiem, J. Jaeck, E. Giard, M. Caes, J.B. Rodriguez, P. Christol, I. Ribet-Mohamed, MTF and FPN measurements to evaluate midwave infrared T2SL focal plane arrays, Proc. SPIE 10111 (2017) $101111 \mathrm{D}$.

[13] D. L. Perry, E.L. Dereniak, Linear theory of nonuniformity correction in infrared staring sensors, Optical Engineering 32 (1993) 1854.

[14] J.M. Mooney, F.D. Sheppard, W.S. Ewing, J.E. Ewing, J. Silverman,Responsivity nonuniformity limited performance of infrared staring cameras, Optical Engineering 28 (1989) 281151.

[15] J.M. Mooney, F.D. Shepherd, Characterizing IR FPA nonuniformity and IR camera spatial noise, Infrared physics \& technology 37 (1996) 595.

[16] A.I. D’Souza, M.G. Stapelbroek, E.W. Robinson, C. Yoneyama, H.A. Mills, M. Kinch, H.D.

Shih, Noise attributes of LWIR HDVIP HgCdTe detectors, Journal of Electronic Materials, 37 (2008) 1318 
[17] A. Brunner, L. Rubaldo, V. Destefanis, F. Chabuel, A. Kerlain, D. Bauza, N.Baier, Improvement of RTS noise in HgCdTe MWIR detectors, Journal of electronic materials 43 (2014) 3060.

[18] D. Pogány, G. Guillot, Random telegraph signal noise instabilities in lattice-mismatched

InGaAs/InP photodiodes, Microelectronics Reliability 39 (1999) 341. 